\title{
An iterative algorithm to solve Algebraic Riccati Equations with an indefinite quadratic term *
}

\author{
Alexander Lanzon \\ Control Systems Centre \\ School of Electrical and Electronic Engineering \\ University of Manchester \\ Sackville Street, Manchester M60 1QD, UK
}

\author{
Yantao Feng $^{\dagger}$ and Brian D. O. Anderson \\ Research School of Information Sciences and Engineering \\ The Australian National University, Canberra ACT 0200, Australia
}

\begin{abstract}
In this paper, an iterative algorithm to solve Algebraic Riccati Equations (ARE) arising from, for example, a standard $H_{\infty}$ control problem is proposed. By constructing two sequences of positive semidefinite matrices, we reduce an ARE with an indefinite quadratic term to a series of AREs with a negative semidefinite quadratic term which can be solved more easily by existing iterative methods (e.g. Kleinman algorithm in [2]). We prove that the proposed algorithm is globally convergent and has local quadratic rate of convergence. Numerical examples are provided to show that our algorithm has better numerical reliability when compared with some traditional algorithms (e.g. Schur method in [5]). Some proofs are omitted for brevity and will be published elsewhere.
\end{abstract}

Keywords: ARE, iterative, indefinite quadratic term

\section{INTRODUCTION}

Consider the following ARE arising from the standard regulator problem:

$$
0=A^{*} P+P A-P B B^{*} P+C^{*} C,
$$

where $A, B, C$ are given real matrices with compatible dimensions, $P$ is the solution sought and $(\cdot)^{*}$ denotes the complex conjugate transpose of $(\cdot)$ here and after. Generally speaking, the existing methods to solve ARE (1) can be divided into two categories:

1. Iterative (see [2] for example): a sequence of matrices

${ }^{*}$ This work has been supported in part by ARC Discovery-Projects Grants DP0342683, DP0664427 and National ICT Australia Ltd. National ICT Australia Ltd. is funded through the Australian Government's Backing Australia's Ability initiative, and in part through the Australian Research Council.

Corresponding author is Alex.Feng@anu.edu.au; tel: +61-2-6125 5891; fax: +61-2-61258660. which converge to the unique stabilizing solution of (1) are constructed.

2. Direct (see [5] for example), based on spectral factorization approach: all solutions of (1) can be constructed from the eigenvectors of the Hamiltonian matrix

$$
H:=\left(\begin{array}{cc}
A & -B B^{*} \\
-C^{*} C & -A^{*}
\end{array}\right) .
$$

When comparing the iterative methods with direct methods, we typically find that iterative methods are numerically more reliable (see [6]).

Now, consider the following ARE arising from standard $H_{\infty}$ control problems

$$
0=\Pi A+A^{*} \Pi-\Pi\left(B_{2} B_{2}^{*}-B_{1} B_{1}^{*}\right) \Pi+C^{*} C,
$$

where $A, B_{1}, B_{2}, C$ are given real matrices with compatible dimensions, and $\Pi \geq 0$ is the unique stabilizing solution sought. Since the Kleinman iterative algorithm is not applicable here due to the non-sign definite quadratic term, a natural question here is: "Is there an iterative algorithm that yields the unique stabilizing solution to ARE (2)?" The answer is positive. In [1], based on an argument using the $L_{2}-$ gain of a given nonlinear control system, the author gives an iterative algorithm to solve the corresponding HamiltonJacobi equations. Although this iterative algorithm to solve Hamilton-Jacobi equations in [1] can be applied to the linear case to solve ARE (2) recursively, it is not easy to execute the iterative algorithm in [1] to solve ARE (2) (or indeed Hamilton-Jacobi equations) since choosing an acceptable initial condition (i.e. $L_{2}$-gain condition) is hard.

In fact, the motivation of this paper comes from the computational disadvantages of current methods to solve ARE (2). In some examples, direct methods (e.g. Schur method) produce very inaccurate solutions (see example 6 in [5]). The reason for such computational inaccuracy is, as pointed out in [5], that there is no guarantee of symmetry for the desired solution $\Pi=U_{21} U_{11}^{-1}$ of $\operatorname{ARE}(2)$ when 
the Schur method is used, where $U_{21}$ and $U_{11}$ are matrices with compatible dimensions which span the stable eigenspace of the Hamiltonian matrix $H$, and $U_{11}$ is invertible. In this paper, we will develop a new iterative algorithm to solve ARE (2) which has computational advantages such as allowing a simple initial condition, high accuracy and high numerical reliability when compared with traditional methods. For example, in our proposed algorithm, we can simply set the initial condition equal zero and then execute our algorithm (this is hence better than the algorithm given in [1]). Furthermore, our proposed algorithm has high numerical reliability compared with the direct methods (e.g. Schur method) (see example 2 in section 6).

In this paper, we reduce the problem of solving a generic Riccati equation with sign indefinite quadratic term to one of generating successive iterations of solutions of LQ-type Riccati equations with negative semidefinite quadratic term (each of which is then amenable to the Newton (Kleinman) algorithm). Consequently, we are reducing a Riccati equation that has no existing iterative scheme to a number of successive iterations of Riccati equations, each of which can be solved by an existing iterative scheme (e.g. Kleinman algorithm). The advantage of doing this is numerical reliability of the calculations involved so as to arrive at the limiting accuracy of the solution (see section 6 for a demonstration of this).

In some sense, we can say that our algorithm is an extension of the Newton (Kleinman) algorithm because it can be applied to solve AREs with an indefinite quadratic term. Furthermore, it has similar advantages as the Newton (Kleinman) algorithm such as high numerical reliability, and local quadratic rate of convergence (see section 5).

The structure of the paper is as follows. Section 2 will establish some preliminary results which will be used in the main theorem. Section 3 will give the main result with a proof of global convergence and section 4 will give the algorithm. Section 5 will give the theorem for the local quadratic rate of convergence. Section 6 will give some numerical examples that demonstrate the effectiveness of our algorithm. Section 7 gives some concluding remarks.

\section{PRELIMINARY RESULTS}

We firstly introduce some notation: Let $\mathbb{R}^{n \times m}$ denote the set of $n \times m$ real matrices; $\mathbb{Z}$ denotes the set of integers with $\mathbb{Z}_{\geq a}$ denoting the set of integers greater or equal to $a \in \mathbb{R} ; \rho[\cdot]$ denotes the spectral radius of a square matrix; $\bar{\sigma}(\cdot)$ denotes the maximum singular value of a matrix; $\operatorname{spec}(\cdot)$ denotes the spectrum of a square matrix. A matrix $A \in \mathbb{R}^{n \times n}$ is said to be Hurwitz if all its eigenvalues have negative real part.

In linear $H_{\infty}$ control, to obtain a suboptimal controller, one needs to solve one or two AREs of the form (2). In such a situation, it is required that the solutions of (2) are not only stabilizing, but also positive semidefinite (see [4]). Since the stabilizing solution to ARE (2) is always unique [11], we seek a unique stabilizing solution $\Pi \geq 0$ to (2) if such solution exists.

The first lemma gathers together some straightforward computations.

Lemma 1 Given real matrices $A, B_{1}, B_{2}, C$ with compatible dimensions, define

$$
\begin{aligned}
F: \mathbb{R}^{n \times n} & \longrightarrow \mathbb{R}^{n \times n} \\
P & \longmapsto P A+A^{*} P-P\left(B_{2} B_{2}^{*}-B_{1} B_{1}^{*}\right) P+C^{*} C .
\end{aligned}
$$

Given also $P=P^{*} \in \mathbb{R}^{n \times n}$ and $Z=Z^{*} \in \mathbb{R}^{n \times n}$, then

$$
F(P+Z)=F(P)+Z \hat{A}+\hat{A}^{*} Z-Z\left(B_{2} B_{2}^{*}-B_{1} B_{1}^{*}\right) Z
$$

where $\hat{A}=A-B_{2} B_{2}^{*} P+B_{1} B_{1}^{*} P$. Furthermore, if $P=P^{*}$, $Z=Z^{*} \in \mathbb{R}^{n \times n}$ satisfy

$$
0=Z \hat{A}+\hat{A}^{*} Z-Z B_{2} B_{2}^{*} Z+F(P),
$$

then

$$
\begin{gathered}
F(P+Z)=Z B_{1} B_{1}^{*} Z \\
\text { and } \quad \rho[F(P+Z)]=\bar{\sigma}\left(B_{1}^{*} Z\right)^{2} .
\end{gathered}
$$

Proof. Equation (4) trivially follows via algebraic multiplications. Results (6) and (7) are simple consequences of (4).

The second lemma sets up some basic relationships between the stabilizing solution $\Pi$ to equation (2) and the matrices $P, Z$ satisfying equation (5).

Lemma 2 Given real matrices $A, B_{1}, B_{2}, C$ with compatible dimensions, $P=P^{*} \in \mathbb{R}^{n \times n}$ and $Z=Z^{*} \in \mathbb{R}^{n \times n}$ satisfying equation (5), and a stabilizing $\Pi=\Pi^{*} \in \mathbb{R}^{n \times n}$ satisfying equation (2), let $\check{A}=A+B_{1} B_{1}^{*}(P+Z)-B_{2} B_{2}^{*} \Pi$ and $\bar{A}=A+B_{1} B_{1}^{*} P-B_{2} B_{2}^{*} \Pi$. Then

(i) $\Pi \geq(P+Z)$ if $\bar{A}$ is Hurwitz,

(ii) $\check{A}$ is Hurwitz if $\Pi \geq(P+Z)$.

Proof. (i) Satisfaction of equation (5) yields satisfaction of equation (6) via Lemma 1 . Adding equation (6) to (2), we have

$F(P+Z)=\Pi A+A^{*} \Pi-\Pi\left(B_{2} B_{2}^{*}-B_{1} B_{1}^{*}\right) \Pi+C^{*} C+Z B_{1} B_{1}^{*} Z$.

Since

$$
\begin{aligned}
F(P+Z)= & (P+Z) A+A^{*}(P+Z) \\
& -(P+Z)\left(B_{2} B_{2}^{*}-B_{1} B_{1}^{*}\right)(P+Z)+C^{*} C
\end{aligned}
$$


via (3), substituting (9) into (8) and rearranging, we have

$$
0=\Sigma \bar{A}+\bar{A}^{*} \Sigma+\Sigma B_{2} B_{2}^{*} \Sigma+(\Pi-P) B_{1} B_{1}^{*}(\Pi-P),
$$

where $\Sigma=\Pi-(P+Z)$. Note that the last two terms in equation (10) are positive semidefinite, so $(i)$ holds by a standard Lyapunov equation type argument (see Lemma 3.18 in [4] for example).

(ii) Rearrange equation (10) as follows:

$$
0=\Sigma \check{A}+\check{A}^{*} \Sigma+\Sigma B_{2} B_{2}^{*} \Sigma+\Sigma B_{1} B_{1}^{*} \Sigma+Z B_{1} B_{1}^{*} Z .
$$

Now letting

$$
W=\left(\begin{array}{c}
B_{2}^{*} \Sigma \\
B_{1}^{*} \Sigma \\
B_{1}^{*} Z
\end{array}\right),
$$

equation (11) becomes

$$
0=\Sigma \check{A}+\check{A}^{*} \Sigma+W^{*} W .
$$

Since $\Pi \geq(P+Z)$ and $W^{*} W \geq 0$, it is only required to show that $(W, \check{A})$ is detectable for the required result to follow (see again Lemma 3.19 in [4] for example). To this end, note that $(W, \check{A})$ is detectable because

$$
\check{A}+L W=A+B_{1} B_{1}^{*} \Pi-B_{2} B_{2}^{*} \Pi
$$

for $L=\left(\begin{array}{lll}0 & B_{1} & 0\end{array}\right)$, which is clearly Hurwitz as $\Pi$ is the stabilizing solution for equation (2). This concludes the proof of part $(i i)$.

\section{MAIN RESULT}

In this section, the main result will be given by constructing two nonnegative matrix sequences $P_{k}$ and $Z_{k}$. The initial condition of our algorithm can be simply set as $P_{0}=0$. We will firstly assert the existence of $P_{k}$ and $Z_{k}$ for $k=0,1,2, \cdots$, and then show that as $k \rightarrow \infty, P_{k}$ converges to the unique stabilizing solution $\Pi$ (which is also positive semidefinite) of ARE (2) if such a solution exists. Under certain assumptions (i.e. $(C, A)$ has no unobservable modes on the $j \omega$-axis, $\left(A, B_{2}\right)$ is stabilizable and provided the unique stabilizing solution $\Pi \geq 0$ to (2) exists), it will be asserted in this section that the stabilizability and detectability conditions needed as the algorithm proceeds are automatically and recursively satisfied.

Theorem 3 Given real matrices $A, B_{1}, B_{2}, C$ with compatible dimensions such that $(C, A)$ has no unobservable modes on the $j \omega$-axis and $\left(A, B_{2}\right)$ is stabilizable, define $F$ : $\mathbb{R}^{n \times n} \longrightarrow \mathbb{R}^{n \times n}$ as in (3). Suppose there exists a stabilizing solution $\Pi$ (which is also positive semidefinite) of ARE (2).

Then
(I) there exist two square matrix series $Z_{k}$ and $P_{k}$ for all $k \in \mathbb{Z}_{\geq 0}$ which satisfy

$$
\begin{gathered}
P_{0}=0 \\
0=Z_{k} A_{k}+A_{k}^{*} Z_{k}-Z_{k} B_{2} B_{2}^{*} Z_{k}+F\left(P_{k}\right), \\
P_{k+1}=P_{k}+Z_{k},
\end{gathered}
$$

where $A_{k}=A-B_{2} B_{2}^{*} P_{k}+B_{1} B_{1}^{*} P_{k} \forall k \in \mathbb{Z}_{\geq 0} ;$

(II) the two series $P_{k}$ and $Z_{k}$ in part (I) can be defined recursively to have the following properties:

1) $\left(A+B_{1} B_{1}^{*} P_{k}, B_{2}\right)$ is stabilizable $\forall k \in \mathbb{Z}_{\geq 0}$,

2) $Z_{k} \geq 0 \forall k \in \mathbb{Z}_{\geq 0}$,

3) $F\left(P_{k+1}\right)=Z_{k} B_{1} B_{1}^{*} Z_{k} \forall k \in \mathbb{Z}_{\geq 0}$,

4) $A+B_{1} B_{1}^{*} P_{k}-B_{2} B_{2}^{*} P_{k+1}$ is Hurwitz $\forall k \in \mathbb{Z}_{\geq 0}$,

5) $\Pi \geq P_{k+1} \geq P_{k} \geq 0 \forall k \in \mathbb{Z}_{\geq 0}$;

(III) let

$$
P_{\infty}:=\lim _{k \rightarrow \infty} P_{k} \geq 0
$$

then $P_{\infty}=\Pi$.

Proof. We construct the series for $Z_{k}$ and $P_{k}$ to show results $(I)$ and $(I I)$ together by an inductive argument. Firstly we show that $(I)$ and $(I I)$ are true for two cases, when $k=0$ and $k=1$ (we need to show two cases because the inductive procedure works on top of the second case, as the conditions for these two cases are different). Then, given $k \in \mathbb{Z}_{\geq 1}$ where $(I)$ and $(I I)$ are satisfied, we will show that $(I)$ and $(I I)$ are also satisfied for $k+1$.

$$
\text { - Case } k=0
$$

Since $P_{0}=0$ via (13), (II1) is trivially satisfied by assumptions. Since equation (14) reduces to

$$
A^{*} Z_{0}+Z_{0} A-Z_{0} B_{2} B_{2}^{*} Z_{0}+C^{*} C=0,
$$

it is standard $[4,10]$ that there exists a unique positive semidefinite stabilizing solution $Z_{0}$ for equation (16), hence $Z_{0} \geq 0$ and (II2) is satisfied. Since $P_{1}=P_{0}+Z_{0}$ via (15), then we have $F\left(P_{1}\right)=Z_{0} B_{1} B_{1}^{*} Z_{0}$ by Lemma 1 and (II3) is satisfied. It is also standard $[4,10]$ that $\left(A-B_{2} B_{2}^{*} Z_{0}\right)$ is Hurwitz (since $Z_{0}$ is the stabilizing solution of (16)), hence (II4) is satisfied on noting that $P_{0}=0$ and $P_{1}=Z_{0}$. We can show (II5) is satisfied by the following steps:

1. Since $Z_{0} \geq 0$ and $P_{1}=P_{0}+Z_{0}$, then $P_{1} \geq P_{0}$;

2. Since $P_{0}=0,\left(A+B_{1} B_{1}^{*} P_{0}-B_{2} B_{2}^{*} \Pi\right)=\left(A-B_{2} B_{2}^{*} \Pi\right)$ is Hurwitz (see [4] or [10]);

3. Since $\left(A+B_{1} B_{1}^{*} P_{0}-B_{2} B_{2}^{*} \Pi\right)$ is Hurwitz, then $\Pi \geq$ $\left(P_{0}+Z_{0}\right)=P_{1}$ by Lemma 2 . 


\section{- Base Case $k=1$}

Note that necessary and sufficient conditions for the existence of a stabilizing solution $Z_{1}$ of equation (14) are (see $[4,10])$ :

(a) $\left(\left(A+B_{1} B_{1}^{*} P_{1}-B_{2} B_{2}^{*} P_{1}\right), B_{2}\right)$ is stabilizable,

(b) $\left(B_{1}^{*} Z_{0},\left(A+B_{1} B_{1}^{*} P_{1}-B_{2} B_{2}^{*} P_{1}\right)\right)$ has no unobservable modes on the $j \omega$-axis;

which equivalently reduce to the following two conditions:

(a1) $\left(A+B_{1} B_{1}^{*} P_{1}, B_{2}\right)$ is stabilizable,

(b1) $\left(B_{1}^{*} Z_{0},\left(A+B_{1} B_{1}^{*} P_{0}-B_{2} B_{2}^{*} P_{1}\right)\right)$ has no unobservable modes on the $j \omega$-axis.

Next we will show that the existence of $Z_{1}$ is guaranteed through the following two points:

1. Since $\Pi \geq P_{1}=\left(Z_{0}+P_{0}\right),\left(A+B_{1} B_{1}^{*} P_{1}-B_{2} B_{2}^{*} \Pi\right)$ is Hurwitz by Lemma 2 . Hence $\left(A+B_{1} B_{1}^{*} P_{1}, B_{2}\right)$ is clearly stabilizable and thus condition $(a 1)$ and (II1) for $k=1$ are satisfied;

2. Since $P_{0}=0$ and $Z_{0}$ is the stabilizing solution of equation (16), $\left(A+B_{1} B_{1}^{*} P_{0}-B_{2} B_{2}^{*} P_{1}\right)=\left(A-B_{2} B_{2}^{*} Z_{0}\right)$ is Hurwitz, hence condition $(b 1)$ is satisfied.

Since $(a 1)$ and $(b 1)$ are satisfied, there exists a unique stabilizing solution $Z_{1} \geq 0$ for equation (14) with $k=1$ (see $[4,10]$ ) on noting that $F\left(P_{1}\right)$ was shown (in the case $k=0$ ) to be $Z_{0} B_{1} B_{1}^{*} Z_{0}$, which is positive semidefinite. So (II2) is satisfied. Since $P_{2}=P_{1}+Z_{1}$ via (15), (II3) is trivially satisfied by Lemma 1 . Since $Z_{1}$ is the stabilizing solution to (14), it follows that $\left(A_{1}-B_{2} B_{2}^{*} Z_{1}\right)=\left(A+B_{1} B_{1}^{*} P_{1}-\right.$ $B_{2} B_{2}^{*} P_{2}$ ) is Hurwitz (see $[4,10]$ ), hence (II4) is satisfied. Since $P_{2}=P_{1}+Z_{1}$ and $Z_{1} \geq 0, P_{2} \geq P_{1}$. Also since we showed earlier (in the case $k=0$ ) that $\Pi \geq P_{1}=\left(P_{0}+Z_{0}\right)$, $\left(A+B_{1} B_{1}^{*} P_{1}-B_{2} B_{2}^{*} \Pi\right)$ is Hurwitz via Lemma 2 Part $(i i)$ and this in turn gives $\Pi \geq P_{2}$ via Lemma 2 Part $(i)$, hence (II5) is satisfied.

\section{- Inductive step for $k \in \mathbb{Z}_{\geq 1}$}

Suppose that for $k=q \in \mathbb{Z}_{\geq 1},(I)$ and (II) are satisfied; we now show that $(I)$ and $(I I)$ are also satisfied for $k=$ $q+1$. Firstly note that necessary and sufficient conditions for the existence of a stabilizing solution $Z_{q+1}$ to (14) are (see $[4,10])$ :

$(\alpha)\left(A_{q+1}, B_{2}\right)$ is stabilizable;

$(\beta)\left(B_{1}^{*} Z_{q}, A_{q+1}\right)$ has no unobservable modes on the $j \omega$ axis.
Conditions $(\alpha)$ and $(\beta)$ are clearly respectively equivalent to the following two conditions:

$(\alpha 1)\left(A+B_{1} B_{1}^{*} P_{q+1}, B_{2}\right)$ is stabilizable;

( $\beta 1)\left(B_{1}^{*} Z_{q}, A+B_{1} B_{1}^{*} P_{q}-B_{2} B_{2}^{*} P_{q+1}\right)$ has no unobservable modes on the $j \omega$-axis.

We will now show the existence of $Z_{q+1}$ is guaranteed via the following two points:

1. Since result $(I I)$ holds for $k=q \in \mathbb{Z}_{\geq 1}$, we have $\Pi \geq$ $P_{q+1}$, and thus $\left(A+B_{1} B_{1}^{*} P_{q+1}-B_{2} B_{2}^{*} \Pi\right)$ is Hurwitz by Lemma 2 Part $(i i)$. Hence $\left(A+B_{1} B_{1}^{*} P_{q+1}, B_{2}\right)$ is stabilizable and thus condition $(\alpha 1)$ and result $(I I)$ for $k=q+1$ are satisfied;

2. Since $\left(A+B_{1} B_{1}^{*} P_{q}-B_{2} B_{2}^{*} P_{q+1}\right)$ was shown to be Hurwitz when $k=q$, condition $(\beta 1)$ is also satisfied.

Since conditions $(\alpha 1)$ and $(\beta 1)$ hold, there exists a unique stabilizing solution $Z_{q+1} \geq 0$ for equation (14) with $k=$ $q+1$ on noting that $F\left(P_{q+1}\right)$ was shown to be positive semidefinite when $k=q$. Hence (II2) is satisfied for $k=$ $q+1$. Since $P_{q+2}=P_{q+1}+Z_{q+1}$, (II3) is trivially satisfied for $k=q+1$ via Lemma 1 . Since $Z_{q+1}$ is the stabilizing solution to (14), it follows that $\left(A_{q+1}-B_{2} B_{2}^{*} Z_{q+1}\right)=(A+$ $\left.B_{1} B_{1}^{*} P_{q+1}-B_{2} B_{2}^{*} P_{q+2}\right)$ is Hurwitz, hence (II4) is satisfied when $k=q+1$. Since $P_{q+2}=P_{q+1}+Z_{q+1}$ and $Z_{q+1} \geq 0$, $P_{q+2} \geq P_{q+1}$. Also, since $\Pi \geq P_{q+1}=P_{q}+Z_{q}$ was shown to hold when $k=q$, it follows that $\left(A+B_{1} B_{1}^{*} P_{q+1}-B_{2} B_{2}^{*} \Pi\right)$ is Hurwitz via Lemma 2 Part (ii) and this in turn gives $\Pi \geq P_{q+2}$ via Lemma 2 Part $(i)$. Hence (II5) is satisfied.

\section{- Inductive Conclusion}

Therefore, together with the cases for $k=0$ and $k=1$ and the inductive step, $(I)$ and $(I I)$ are true $\forall k \in \mathbb{Z}_{\geq 0}$, hence the proof for $(I)$ and $(I I)$ is completed.

(III) Since the sequence $P_{k}$ is monotone and bounded above by $\Pi$, the sequence converges to a limit $P_{\infty}$ (see [9]), and convergence of the sequence $P_{k}$ to $P_{\infty}$ implies convergence of $Z_{k}$ to 0 since

$$
Z_{\infty}:=\lim _{k \rightarrow \infty} Z_{k}=\lim _{k \rightarrow \infty}\left(P_{k+1}-P_{k}\right)=0 .
$$

Then it is clear from (II5) that $P_{\infty} \geq 0$, from (II3) that $P_{\infty}$ must satisfy $F\left(P_{\infty}\right)=0$, and from (II4) that $P_{\infty}$ must satisfy $\left(A+B_{1} B_{1}^{*} P_{\infty}-B_{2} B_{2}^{*} P_{\infty}\right)$ is Hurwitz. Thus $P_{\infty} \geq 0$ is a stabilizing solution to $F\left(P_{\infty}\right)=0$. Since $\Pi \geq 0$ is the unique stabilizing solution of $F(\Pi)=0$, it is clear that $P_{\infty}=\Pi($ see $[11])$.

The following corollary gives a condition under which there does not exist a stabilizing solution $\Pi \geq 0$ to $F(\Pi)=$ 0 . This is useful for terminating the recursion in finite iterations. 
Corollary 4 Given real matrices $A, B_{1}, B_{2}, C$ such that $(C, A)$ has no unobservable modes on the $j \omega$-axis and $\left(A, B_{2}\right)$ is stabilizable, define $F: \mathbb{R}^{n \times n} \longrightarrow \mathbb{R}^{n \times n}$ as in (3). If $\exists k \in \mathbb{Z}_{\geq 0}$ such that $\left(A+B_{1} B_{1}^{*} P_{k}, B_{2}\right)$ is not stabilizable, where $P_{k}$ arises from iterating (13)-(15) and as defined in Theorem 3 Part (II), then there does not exist a stabilizing solution $\Pi \geq 0$ to $F(\Pi)=0$.

Proof. Restatement of Theorem 3, implication (II1).

\section{ALGORITHM}

Given real matrices $A, B_{1}, B_{2}, C$ with compatible dimensions and supposing $(C, A)$ has no unobservable modes on the $j \omega$-axis and $\left(A, B_{2}\right)$ is stabilizable, an iterative algorithm for finding the positive semidefinite stabilizing solution of equation (2) is given as follows:

1. Let $P_{0}=0$ and $k=0$.

2. Construct (for example using the Kleinman algorithm in [2], though this is not necessary) the unique real symmetric stabilizing solution $Z_{k} \geq 0$ which satisfies

$$
0=Z_{k} A_{k}+A_{k}^{*} Z_{k}-Z_{k} B_{2} B_{2}^{*} Z_{k}+F\left(P_{k}\right),
$$

where $A_{k}=A-B_{2} B_{2}^{*} P_{k}+B_{1} B_{1}^{*} P_{k}$ and $F\left(P_{k}\right)$ is given in (3).

3. Set $P_{k+1}=P_{k}+Z_{k}$.

4. If $\bar{\sigma}\left(B_{1}^{*} Z_{k}\right)<$ a specified accuracy, then set $\Pi=P_{k+1}$ and exit. Otherwise, go to step 5.

5. If $\left(A+B_{1} B_{1}^{*} P_{k+1}, B_{2}\right)$ is stabilizable, then increment $k$ by 1 and go back to step 2 . Otherwise, exit as there does not exist a real symmetric stabilizing solution $\Pi \geq 0$ satisfying $F(\Pi)=0$.

From Corollary 4 we see that if the stabilizability condition in step 5 fails at some $k \in \mathbb{Z}_{\geq 0}$, then there does not exist a stabilizing solution $\Pi \geq 0$ to $F(\Pi)=0$ and the algorithm should terminate (as required by step 5 ). But when this stabilizability condition is satisfied $\forall k \in \mathbb{Z}_{\geq 0}$, construction of the series $P_{k}$ and $Z_{k}$ is always possible and either $P_{k}$ converges to $\Pi$ (which is captured by step 4 ) or $P_{k}$ just diverges to infinity, which again means that there does not exist a stabilizing solution $\Pi \geq 0$ to $F(\Pi)=0$.

\section{RATE OF CONVERGENCE}

As mentioned in the introduction section, our algorithm, in some sense, is an extension of the Kleinman algorithm in [2] since we solve ARE (2) iteratively and the Kleinman algorithm solves ARE (1) iteratively. (Of course ARE (2) is identical to $\operatorname{ARE}(1)$ when $B_{1}=0$ ). In this section, we claim, without proof, a local quadratic rate of convergence for the algorithm given in section 4 . The proof will be published elsewhere in an extended treatment.

Theorem 5 Given the suppositions of Theorem 3, and with the two series $P_{k}, Z_{k}$ as defined in Theorem 3 Part II, the rate of convergence of the series $P_{k}$ in the vicinity of $\Pi$ is quadratic.

Proof. This will be published elsewhere.

\section{NUMERICAL EXAMPLES}

In this section, three examples are given. Example 1 provides a numerical comparison with other existing approaches, and shows that our algorithm works well when other approaches work well too. Example 2 shows that our algorithm still works well when other approaches (such as the MATLAB command CARE and the Schur method of [5]) do not work. Example 3 demonstrates that there in fact does not exist a stabilizing solution $\Pi \geq 0$ to equation (2) when the stabilizability condition in step 5 of the algorithm is not satisfied.

\subsection{Example 1}

This simple example is used to illustrate that the algorithm proposed in this paper works well in situations where the traditional MATLAB command CARE also works well. Let

$$
\begin{gathered}
A=\left(\begin{array}{cccc}
0.4012 & 0.6905 & -0.9266 & 0.9514 \\
-0.4073 & -1.8646 & 0.0948 & -0.2067 \\
0.1834 & -1.1192 & -0.0401 & -0.3414 \\
-0.4361 & -3.3770 & 0.5495 & -1.3014
\end{array}\right), \\
B_{1}=\left(\begin{array}{ccc}
-0.4784 & -1.9013 & -0.0002 \\
-0.7406 & 0.6155 & -1.1518 \\
-0.4175 & -0.4381 & -1.8218 \\
0.6643 & -0.9887 & -0.5926
\end{array}\right), B_{2}=\left(\begin{array}{ccc}
0.1673 & -1.2535 \\
2.3069 & 0.1293 \\
-0.5521 & 0.7904 \\
-1.3909 & 2.1308
\end{array}\right), \\
C=\left(\begin{array}{cccc}
-0.8627 & -1.5309 & 0.6475 & 1.0916 \\
-0.6507 & 0.6614 & 0.1981 & -0.0948
\end{array}\right) .
\end{gathered}
$$

The traditional MATLAB command CARE gives the solution:

$$
\Pi=\left(\begin{array}{cccc}
1.2556 & 0.0358 & -0.8109 & 0.4724 \\
0.0358 & 0.5091 & -0.0435 & -0.3036 \\
-0.8109 & -0.0435 & 2.3613 & -0.9541 \\
0.4724 & -0.3036 & -0.9541 & 0.6858
\end{array}\right)
$$

The algorithm proposed in this paper gives an almost identical solution after ONLY 2 iterations. The norm of the error between the two solutions is $1.2831 \times 10^{-4}$.

\subsection{Example 2}

The following example illustrates that the proposed algorithm works well when other traditional methods fail. This example is a slight modification of example 6 in [5]. 
Choose the matrix $A \in \mathbb{R}^{21 \times 21}, B_{1} \in \mathbb{R}^{21 \times 1}, B_{2} \in \mathbb{R}^{21 \times 1}, C \in$ $\mathbb{R}^{21 \times 21}$ in equation (2) as follows:

$A=\left(\begin{array}{ccccc}0 & 1 & 0 & \cdots & 0 \\ & \ddots & \ddots & 0 & \vdots \\ \vdots & & \ddots & \ddots & 0 \\ & & & \ddots & 1 \\ 0 & 0 & \ldots & & 0\end{array}\right), B_{1}=\left(\begin{array}{c}0 \\ \vdots \\ 0 \\ \delta \\ 0\end{array}\right), B_{2}=\left(\begin{array}{c}0 \\ \vdots \\ 0 \\ 0 \\ 1\end{array}\right)$,

$C=\operatorname{diag}\{1,0, \cdots, 0\}$, where $\delta=10^{-2}$ is the introduced modification. In this example, the Schur method in [5] does not produce an accurate result, similarly to the MATLAB command CARE. The algorithm proposed by Kleinman in [2] cannot be used because the term $\left(B_{2} B_{2}^{*}-B_{1} B_{1}^{*}\right)$ in Riccati equation (2) is not positive semidefinite. However, the algorithm proposed in the paper easily gives the solution with the specified accuracy as will be shown next.

Firstly we attempt the Schur method in [5] with this example. Let $F$ be defined as in (3), and $S_{1}$ be the solution to equation (2) by using this Schur method. We evaluate the accuracy of the solution $S_{1}$ by calculating $\rho\left[F\left(S_{1}\right)\right]$. The smaller $\rho\left[F\left(S_{1}\right)\right]$ is, the closer $S_{1}$ is to the correct solution. After calculation, we obtain $\rho\left[F\left(S_{1}\right)\right]=1.9802 \times 10^{3}$ which is far too large. Thus, we can conclude that the Schur method in [5] fails to give a solution in this example. Similarly, let $S_{2}$ be the solution obtained by the MATLAB command CARE. For this solution, we can obtain $\rho\left[F\left(S_{2}\right)\right]=$ $1.9811 \times 10^{3}$ which again is too large. So we conclude that MATLAB command CARE also fails to give a solution in this example. If we were to try to refine the very coarse solution obtained by the Schur method in [5] using Kleinman's method in [2], this too fails as this algorithm diverges with each iteration (as expected). This can be shown as follows: let $X_{k}$ with $k \in \mathbb{Z}_{\geq 1}$ denote the iterative series produced by the Kleinman algorithm, then we obtain $\rho\left[F\left(X_{1}\right)\right]=5.7083 \times 10^{2}, \rho\left[F\left(X_{2}\right)\right]=5.9959 \times 10^{2}, \cdots$, $\rho\left[F\left(X_{20}\right)\right]=8.2965 \times 10^{7}, \cdots, \rho\left[F\left(X_{100}\right)\right]=6.6206 \times 10^{9}$.

However, when we use our proposed algorithm, we note that a unique stabilizing solution $P_{4}>0$ to equation (2) can be found with limiting accuracy after only 4 iterations with $\rho\left[F\left(P_{4}\right)\right]=\bar{\sigma}\left(B_{1}^{*} Z_{3}\right)^{2}=2.9205 \times 10^{-5}$.

\subsection{Example 3}

The following example shows that if $\left(A+B_{1} B_{1}^{*} P_{k+1}, B_{2}\right)$ is not stabilizable in step 5 of the algorithm, then there does not exist a stabilizing solution $\Pi \geq 0$ to equation (2). Choose

$$
\begin{gathered}
A=\left(\begin{array}{cc}
1 & 100 \\
1 & 0
\end{array}\right), B_{1}=\left(\begin{array}{c}
10 \\
0
\end{array}\right), \\
B_{2}=\left(\begin{array}{l}
0 \\
1
\end{array}\right), C=\left(\begin{array}{ll}
1 & 0
\end{array}\right) .
\end{gathered}
$$

We note that $(C, A)$ has no unobservable modes on the $j \omega$-axis and $\left(A, B_{2}\right)$ is stabilizable. When we run our algorithm, we find that $\left(A+B_{1} B_{1}^{*} P_{1}, B_{2}\right)$ is not stabilizable after one iteration since

$$
P_{1}=\left(\begin{array}{cc}
0.3517 & 2.6442 \\
2.6442 & 22.9964
\end{array}\right) \text {. }
$$

This is consistent with the fact that there does not exist a stabilizing solution $\Pi \geq 0$ to equation (2). In fact, we can find the unique stabilizing solution

$$
\Pi=\left(\begin{array}{cc}
-0.5744 & -4.9147 \\
-4.9147 & -37.8481
\end{array}\right),
$$

which is clearly not positive semidefinite.

\section{CONCLUSIONS}

In this paper, we proposed an iterative algorithm to solve AREs arising from standard $H_{\infty}$ control problems. Numerical examples have been provided to show that our algorithm has superior numerical reliability when compared with other existing methods. Furthermore, we have also proved that our algorithm has global convergence, can be initialized with a simple choice $P_{0}=0$, and we claimed a local quadratic rate of convergence. It can hence be anticipated that our algorithm can be used in situations where AREs need to be solved with both high speed and high accuracy.

\section{References}

[1] A.J. van der Schaft, " $L_{2}$-gain analysis of nonlinear systems and nonlinear systems and nonlinear state feedback $H_{\infty}$ control”, IEEE Trans. on Automatic Control, 37 (1992), pp. 770-784.

[2] D. L. Kleinman. "On an iterative technique for Riccati equation computation", IEEE Trans. on Automatic Control, vol. 13, pp. 114-115, 1968.

[3] W. M. Wonham. "On a matrix Riccati equation of stochastic control ”, SIAM J. Control, Vol.6, No.4 pp 681-697, 1968.

[4] K. Zhou with J. C. Doyle and K. Glover, Robust and optimal control, Prentice Hall, Upper Saddle River, NJ; 1996.

[5] A. J. Laub, "A Schur method for solving algebraic Riccati equation", IEEE Trans. on Automatic Control, vol. 24, pp. 913-921, 1979.

[6] W. F. Arnold, III and A. J. Laub, "Generalized eigenproblem algorithms and software for algebraic Riccati equations", Proceedings of the IEEE, vol. 72, Issue 12, pp.1746-1754, 1984.

[7] G. H. Golub and C. F. Van Loan, Matrix Computations, The John Hopkins University Press, Baltimore and London; 1996.

[8] R. H. Bastels and W. Stewart, "Solution of the matrix $A X+$ $X B=C$ ", Communications of ACM, vol.15, pp 820-826, 1972. 
[9] H. J. Wilcox and D. L. Myers, An Introduction to Lebesgue Integration and Fourier Series, Courier Dover Publications, 1995.

[10] M. Green and D. J. N. Limebeer, Linear robust control, Prentice Hall, Englewood Cliffs, NJ; 1995.

[11] J. C. Willems, "Least Squares Stationary Optimal Control and the Algebraic Riccati Equation", IEEE Trans. on Automatic Control, vol. AC-16, NO.6, pp. 621-634, 1971. 